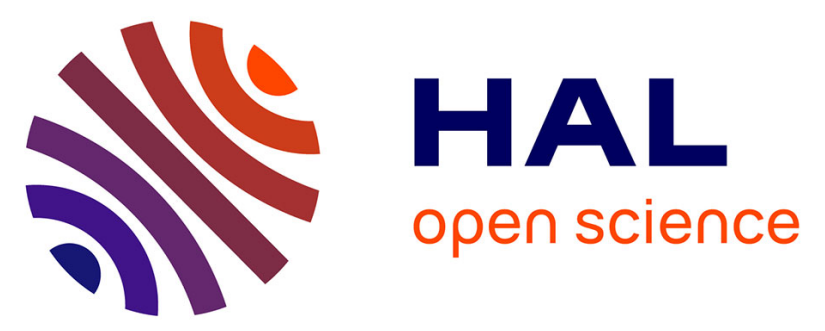

\title{
Comparison between cohesive zone and coupled criterion modeling of crack initiation in rhombus hole specimens under quasi-static compression
}

Aurélien Doitrand, Rafael Estevez, Dominique Leguillon

\section{- To cite this version:}

Aurélien Doitrand, Rafael Estevez, Dominique Leguillon. Comparison between cohesive zone and coupled criterion modeling of crack initiation in rhombus hole specimens under quasi-static compression. Theoretical and Applied Fracture Mechanics, 2019, 99, pp.51-59. 10.1016/j.tafmec.2018.11.007 . hal-02173117

\section{HAL Id: hal-02173117 \\ https: / hal.sorbonne-universite.fr/hal-02173117}

Submitted on 4 Jul 2019

HAL is a multi-disciplinary open access archive for the deposit and dissemination of scientific research documents, whether they are published or not. The documents may come from teaching and research institutions in France or abroad, or from public or private research centers.
L'archive ouverte pluridisciplinaire HAL, est destinée au dépôt et à la diffusion de documents scientifiques de niveau recherche, publiés ou non, émanant des établissements d'enseignement et de recherche français ou étrangers, des laboratoires publics ou privés. 


\title{
Comparison between cohesive zone and coupled criterion modeling of crack initiation in rhombus hole specimens under quasi-static compression
}

\author{
Aurélien Doitrand $^{1 *}$, Rafael, Estevez ${ }^{1}$, Dominique Leguillon ${ }^{2}$ \\ ${ }^{1}$ Université Grenoble-Alpes - CNRS UMR 5266, SIMaP, F-38000 Grenoble, France \\ ${ }^{2}$ Institut Jean le Rond d'Alembert, Sorbonne Université, Centre National de la Recherche Scientifique, UMR 7190, F-75005 \\ Paris, France \\ dominique.leguillon@upmc.fr
}

\begin{abstract}
Numerical predictions of initiation loading and crack length in a rhombus hole specimen made of a brittle material under quasi-static compression using either cohesive zone model or coupled criterion based on finite fracture mechanics are compared. Both methods lead to the conclusion that crack initiation results in a crack jump over a finite length at a given loading level, which depends on the material parameters and on the specimen geometry. The initiation load level and crack extent depend on the cohesive zone traction-opening displacement profile which represents the underlying failure mechanism. The ranges of initiation force and crack lengths obtained with various traction-opening profiles respectively comprise the initiation force and lower bound for crack arrest derived from the coupled criterion. For the configuration under investigation, similar initiation forces and crack arrest lengths as those predicted using the coupled criterion are obtained using a bilinear cohesive zone model.
\end{abstract}

Keywords:

Cohesive zone, Coupled criterion, Crack initiation

\section{Introduction}

In a industrial design process, crack nucleation and growth determination based on predictive modeling tools appears to be a main challenge so as to reduce costly and time consuming experiments. Classical linear elastic fracture mechanics allows the growth of a pre-existing crack to be studied but fails to predict crack nucleation. To this aim, approaches based on Finite Fracture Mechanics (FFM) were developed so as to study finite crack increments $[18,34]$. In this context, the abrupt nucleation a crack over a finite length can be modeled by the so-called Coupled Criterion (CC) initially proposed by Leguillon [20]. This approach is applicable for crack initiation prediction in various configurations (V-notch [7, 22, 25], interfaces $[27,28,30,38]$, etc) and bulk materials (composites [9, 10, 17, 31], ceramics [24, 26], etc). An extensive review of the CC applications was recently proposed by Weißgraeber et al. [41].

Another approach that is usually employed in order to describe material or structure failure is Cohesive Zone Model (CZM) $[2,4,13,39,40,43]$ that consists in defining a traction-opening relationship that mimicks

\footnotetext{
*corresponding author, aurelien.doitrand@grenoble-inp.fr
} 
mechanically the failure process. CZM describes the relation between the cohesive stress and the displacement jump between both surfaces. When the traction reaches a critical value, progressive debonding takes place up to a critical opening corresponding to the local nucleation of a crack. A main difference between CZM and $\mathrm{CC}$ is the definition of a process zone between undamaged and completely failed zones. Nevertheless, both approaches basically require the same input data, namely the use of (at least) two failure parameters: a critical traction $\sigma_{\mathrm{c}}$ and a critical energy release rate $G^{\mathrm{c}}$. CC and CZM can be employed in order to model the same fracture problem, which allows comparing both approaches.

Several authors compared crack initiation prediction using CC and CZM. For instance, Cornetti et al. [7] predicted similar initiation loading levels for both models in the case of short cracks and V-notch. Both methods were also employed for modeling fiber/matrix interface cracking in composite materials [15, 37]. Poitou et al. [37] obtained similar initiation strains using either Dugdale model [13] or CC. García et al. [15] showed different degrees of agreement between both models, depending on some geometric features such as the fiber radius size. They also highlighted the fact that CC predicts an abrupt initiation on a finite length whereas CZM is based on a progressive debonding. However, Henninger et al. [19] demonstrated that either CC or CZM result in crack initiation being forthwith followed by an unstable crack propagation that should lead the crack to directly jump to a finite length, but no details were given about this unstable crack propagation following nucleation. The spontaneity of crack nucleation was also discussed by Martin et al. [32] in the case of edge debonding. Similar predictions of crack initiation level and load-displacement curve between CC and CZM were obtained provided that the characteristic fracture length of the material remained small enough compared to a characteristic length of the studied configuration. Otherwise, noticeable differences were observed since using CZM led to a large process zone and thus postponed crack initiation compared to CC. Cornetti et al. [8] recently showed that the loading level predicted by FFM and CZM could even match in the case of large process zones, which was achieved by introducing weight functions in the stress condition of the CC. Most of the previously cited works focused on comparing the initiation loading level obtained using CC or CZM but include few analysis about the initiation crack length.

The objective of the present work is to provide additional insight about the comparison between CC and CZM approach for crack initiation prediction, especially concerning the spontaneous nature of crack nucleation, as well as the resulting crack length. The studied configuration is rhombus hole specimens under compression, which are chosen because they lead to a crack arrest after initiation, followed by a stable crack propagation. The CC is described in Section 2 and the CZM in Section 3. Numerical considerations for both

methods are discussed in section 4 and the numerical prediction of crack initiation using both methods are compared in Section 5 .

\section{Coupled criterion}

\subsection{Crack initiation determination}

The initiation of a crack under quasi-static loading requires two separate conditions to be simultaneously fulfilled [20]. The first requirement is a high enough stress state over the whole expected crack surface prior 
to crack nucleation. For an isotropic homogeneous material, this condition consists in comparing the stress normal to the crack plane to the material strength (Eq. 1)

$$
\sigma_{\mathrm{nn}} \geqslant \sigma_{\mathrm{c}} \text { over the whole expected crack surface. }
$$

The second requirement is derived from a balance, between an elastic state before and after crack initiation, of the potential energy $\left(\delta W_{p}\right)$, the kinetic energy $\left(\delta W_{k}\right)$ and the energy dissipated by crack nucleation $\left(G_{c} S\right.$, $G_{c}$ being the material toughness and $S$ the newly created crack surface). Under quasi-static initial conditions, there is a production of kinetic energy $\left(\delta W_{k}>0\right)$, which leads to the energy condition that compares the incremental energy release rate, i.e. the ratio between the energy dissipated by the crack opening (which corresponds to the change in potential energy $\Delta W(S)=W(0)-W(S))$ and the surface $S$ of the crack, to the material critical energy release rate $G^{\text {c }}$ (Eq. 2),

$$
G^{\mathrm{inc}}(S)=-\frac{\Delta W(S)}{S}=\frac{W(0)-W(S)}{S} \geq G^{\mathrm{c}} .
$$

Under the assumption of linear elasticity and small deformation, the stress is proportional to the applied load $P$ whereas the potential energy of both the damaged and the undamaged material, and hence the incremental energy release rate, are proportional to the square of applied load (Eq. 3). In practice, only one calculation is required in order to compute $k(S)$ and hence the stress criterion. Several calculations with varying crack surface allow $A(S)$ and thus the energy criterion to be computed,

$$
\left\{\begin{array}{l}
\sigma_{\mathrm{nn}}(S)=k(S) P=\sigma_{\mathrm{c}} \\
G^{\mathrm{inc}}=A(S) P^{2}=G^{\mathrm{c}}
\end{array}\right.
$$

The crack configuration at damage initiation (i.e. $S^{*}$ ) can be determined by solving Eq. 4, as the configuration for which both the energy and the stress conditions are satisfied for a minimum imposed loading $P^{*}[11,20]$, as

$$
\frac{A\left(S^{*}\right)}{k\left(S^{*}\right)^{2}}=\frac{G^{\mathrm{c}}}{\sigma_{\mathrm{c}}^{2}}
$$

\subsection{Stability of the crack at initiation}

The coupled criterion allows the determination of finite crack nucleation configuration, i.e. a crack surface $S^{*}$ at a prescribed loading $P^{*}$. The growth of the nucleated crack can then be studied considering the differential energy release rate $G=-\frac{d W}{d S}$, which can be expressed as a function of the incremental energy release rate, its derivative and the crack surface (Eq. 5).

$$
G(S)=-\frac{d W(S)}{d S}=\frac{d G^{\mathrm{inc}}(S)}{d S} S+G^{\mathrm{inc}}(S)
$$

It is worth noting that since $G^{\text {inc }}$ has already been computed to determine the nucleation configuration, no extra calculations are needed to compute $G$ which is obtained using Eq. 5. After crack nucleation, several propagation scenarii may happen depending on the differential energy release rate:

o No propagation : $G<G^{\mathrm{c}}$, 
o Stable propagation: $G=G^{\mathrm{c}}$ and $\frac{d G}{d S} \leqslant 0$,

o Unstable propagation: $G>G^{\mathrm{c}}$ or $\left(G=G^{\mathrm{c}}\right.$ and $\left.\frac{d G}{d S} \geqslant 0\right)$.

In the case of stable propagation, an increase in the loading is required so that the crack can grow, whereas an unstable crack propagation is achieved without any increase in the loading. At crack initiation, $G^{\text {inc }}=G^{\mathrm{c}}$, therefore the stability of the propagation depends on the $\operatorname{sign}$ of $\frac{d G^{\mathrm{inc}}(S)}{d S}$, i.e. on the monotony of the incremental energy release rate. A strictly increasing monotonic variation of $G^{\text {inc }}$ leads to an unstable propagation of the crack just after initiation which may even trigger the specimen failure [11, 12]. Fig. 1 shows the case of a non monotonic variation of $G^{\text {inc }}$, i.e. $G^{\text {inc }}$ attains a maximum for a crack surface $S_{\mathrm{m}}[9,29,42]$. In most cases, crack initiation surface verifies $S^{*}<S_{\mathrm{m}}$ (Fig. 1) so that $\frac{d G^{i n c}}{d S}>0$ and $G>G^{\text {inc }}=G^{\text {c }}$, which leads to an unstable propagation of the crack. The crack will propagate at least up to a surface $S_{\min }$ satisfying $G\left(S_{\min }\right)=G^{\mathrm{c}}$. However, during crack propagation from $S^{*}$ to $S_{\min }$, the released energy is higher than the energy required for the crack growth $\left(G>G^{\mathrm{c}}\right)$. This energy excess may be consumed for further crack propagation up to a surface $S_{\max }$ verifying Eq. 6 . Besides, it can be shown that Eq. 6 is equivalent to $G^{\text {inc }}\left(S_{\max }\right)=G^{\text {c }}$.

$$
\int_{S^{*}}^{S_{\min }}\left(G(S)-G^{\mathrm{c}}\right) d S=\int_{S_{\min }}^{S_{\max }}\left(G^{\mathrm{c}}-G(S)\right) d S
$$

In practice, only a part of the energy excess is consumed for further crack propagation while the other part may be dissipated [42]. Since it is really difficult to quantify the ratio between the energy excess that is dissipated or used for further crack propagation, the crack surface observed experimentally may be compared to the lower $\left(S_{\min }\right.$ verifying $\left.G\left(S_{\min }\right)=G^{\mathrm{c}}\right)$ and upper $\left(S_{\max } \operatorname{verifying} G^{\mathrm{inc}}\left(S_{\max }\right)=G^{\mathrm{c}}\right)$ bound estimates of the crack arrest surface. A particular case concerns crack initiation surface corresponding to the maximum of the incremental energy release rate $\left(S^{*}=S_{\mathrm{m}}\right)$ (Fig. 1), leading to $\frac{d G^{\text {inc }}}{d S}=0$ and $G=G^{\text {inc }}=G^{\mathrm{c}}$. Therefore,

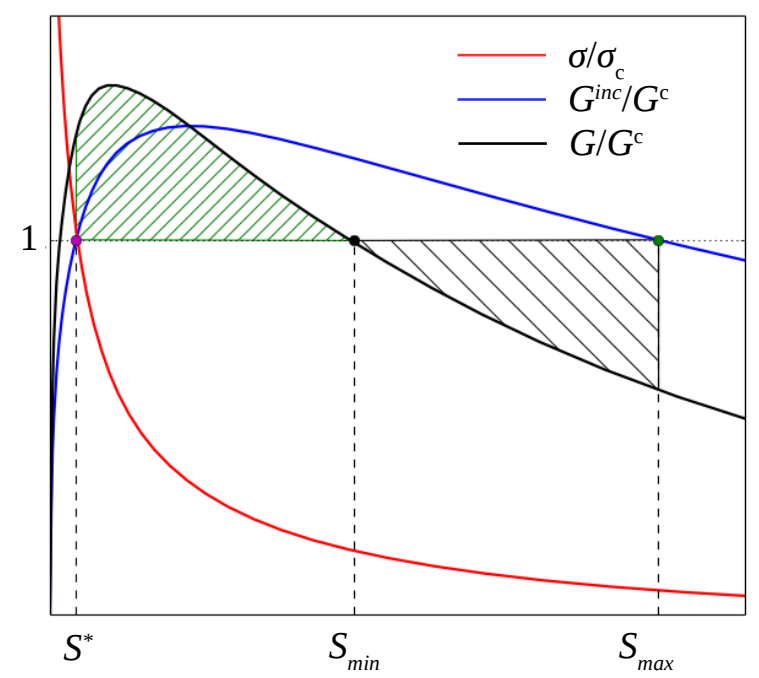

- Initiation configuration

- Lower bound for crack arrest

- Upper bound for crack arrest

Excess energy not consumed during unstable crack propagation

Available energy that can possibly be used for further crack propagation

Figure 1: Stress to strength, incremental and differential energy release rate to toughness ratios as a function of the crack surface. Crack initiation $\left(S=S^{*}\right)$ is followed by unstable crack propagation up to an arrest surface comprised between $S_{\min }$ and $S_{\max }$. 
the crack does not propagate without any increase in the loading, which means that the initiation crack surface is also an arrest surface $\left(S^{*}=S_{\min }=S_{\max }\right)$. In this case, the crack initiation surface predicted with the $\mathrm{CC}$ can directly be compared to that observed experimentally.

\section{Cohesive zone model}

A cohesive zone model defines a relation between the cohesive traction $T$ and the opening displacement $\delta$ of a process zone. It is usually composed of a first part corresponding to an elastic undamaged behavior, for which the traction increases up to a critical value $\sigma_{\mathrm{c}}$ corresponding to a characteristic opening displacement $\delta_{0}$. When the traction has reached $\sigma_{\mathrm{c}}$, debonding occurs with a decrease in the cohesive traction until a critical opening displacement $\delta_{\mathrm{c}}$ for which the traction becomes null, hence corresponding to the nucleation of a crack locally. The area under the traction-opening profile correspond to the work for separation and creation of free surfaces, equal to $G^{\mathrm{c}}$ for an elastic material with no dissipation effects. Fig. 2a shows an example of traction-opening displacement curve in the case of a bilinear cohesive zone model. In addition to $G^{\mathrm{c}}, \sigma_{\mathrm{c}}$ and $\delta_{\mathrm{c}}$, the traction-opening profile can be different depending on the underlying failure mechanism $[2,3,13,35,40,43]$. Alfano [3] compared several cohesive models and concluded that the traction-opening profile can influence the response of the studied configuration, depending on the boundary value problem. Moreover, Alfano showed that this influence is only noticeable in the vicinity of the maximum applied load (i.e., at crack initiation in the studied configuration). This conclusion is in agreement with that of Acary and Monerie [1] who showed that all the cohesive zone models are equivalent for studying stable rectilinear crack propagation but not for crack initiation and branching. The influence of the traction-opening displacement profile on crack initiation is studied in Section 4.3 by employing the model of Park et al. [35, 36] which enables different traction-opening displacement profiles to be obtained by varying a single shape parameter (for fixed fracture parameters $G^{\mathrm{c}}$ and $\sigma_{\mathrm{c}}$ ). For the configuration under investigation in this study, cracking occurs in mode I. Therefore, mode mixity, which can also be taken into account using CC [5, 16, 32] or CZM $[2,3,35]$, will not be considered in this work. Contrary to the CC presented in Section 2 for which

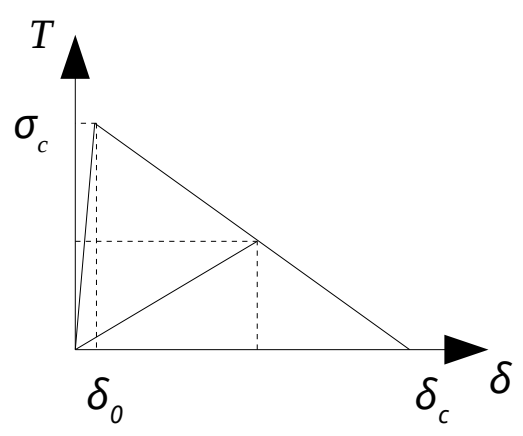

(a)

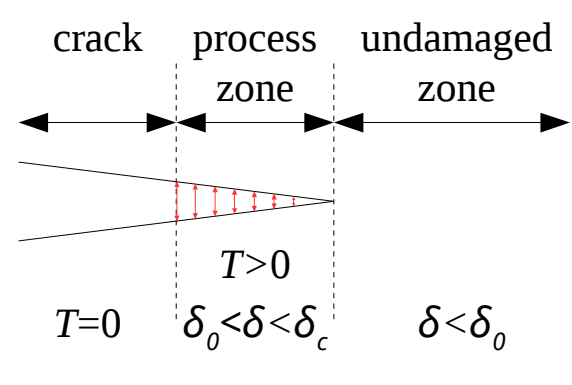

(b)

Figure 2: (a) Traction-opening displacement curve (example of bilinear model) and (b) schematic representation of the process zone. 


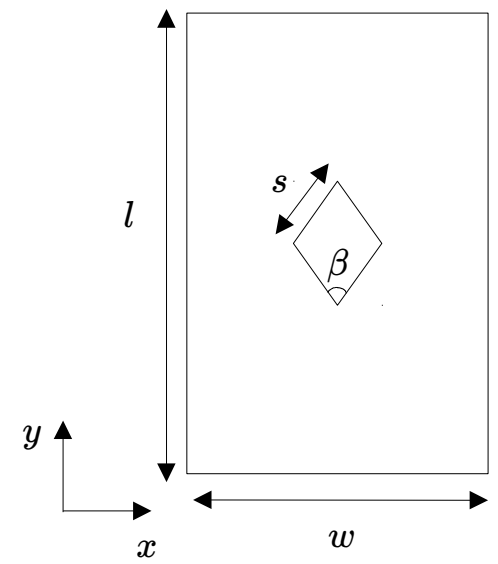

(a)

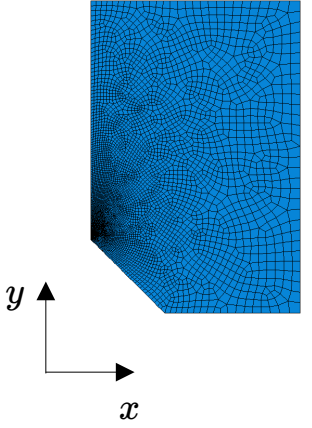

(b)

Figure 3: (a) Geometry of the rhombus hole specimen and (b) mesh of a quarter of specimen.

the definition of a crack is straightforward, the definition of a cohesive zone model introduces the concept of process zone (cf. Fig. 2b). In the following, the crack length for CZM is defined as the traction-free zone which does not include the process zone ( $c f$. Fig. 2b). The initiation loading is therefore defined as the minimum loading for which a crack nucleates (i.e. $\delta=\delta_{c}$ and $T=0$ in the CZM).

\section{Numerical implementation of CC and CZM}

\subsection{Finite Element models}

The configuration chosen for the comparison of CC and CZM is quasi-static compression of rhombus hole specimen, whose geometry is depicted in Fig. 3a. A uniaxial compression in the y-direction is generated by imposing a vertical displacement on the specimen top edge. Under such a loading, a mode I vertical crack initiates at the rhombus hole upper and lower V-notch due to the lateral expansion. The symmetric configuration allows to model only one specimen quarter, for which a mesh example is presented in Fig. 3b. The specimen length and width respecively are $l=60 \mathrm{~mm}$ and $w=40 \mathrm{~mm}$. The mechanical properties chosen for both CC and CZM computation are $E=1600 \mathrm{GPa}, \nu=0.37, \sigma_{\mathrm{c}}=80 \mathrm{MPa}$ and $G^{\mathrm{c}}=250 \mathrm{~J} / \mathrm{m}^{2}$, which are representative of PMMA. All the calculations presented in this work have been performed in 2D using Abaqus standard v6.13. Since only $2 \mathrm{D}$ models are considered in this work, in the following, the crack is described by its length $L$ rather than its surface $S$. The initiation crack length (solution of Eq. 4) is noted $L^{*}$, and the crack arrest length $L^{\text {arrest }}$.

\subsection{CC implementation}

The implementation of the $\mathrm{CC}$ for crack initiation prediction in rhombus hole specimens requires both the stress and the energy conditions described in Section 2 to be computed. This is done by FE calculations on undamaged and damaged configurations of rhombus hole specimens, whose dimensions are depicted in Fig. 
3a. The stress criterion is computed from a calculation on undamaged specimen meshed with 4 node elements (Fig. 3b). The energy criterion is obtained by computing the potential energy of both the undamaged and damaged configuration for several crack lengths, which requires the generation of the corresponding crack meshes. In 2D, the crack is only defined by one parameter, namely its length $L$ along $(O y)$-axis so that 2D cracked mesh generation is straightforward by doubling the nodes on the presupposed crack path. Once the stress and the energy conditions have been computed, the method presented in Section 2 is applied in order to determine the crack initiation and arrest lengths, as well as the force at initiation.

Fig. 4 and Table 1 show the incremental energy release rate and the initiation force and crack arrest lengths predicted by the CC using different mesh refinement levels along the crack path in the case of a hole with $\beta=90$ deg. angle and $s \times \cos \left(\frac{\beta}{2}\right)=4.95 \mathrm{~mm}$ half-diagonal. The number of calculations corresponds to the number of nodes that are successively doubled in order to compute the energy release rate as a function of the crack length.

\begin{tabular}{|c|c|c|c|c|c|c|}
\hline maximum mesh size $(\mathrm{mm})$ & 2. & 1. & 0.5 & 0.5 & 0.5 & 0.5 \\
\hline minimum mesh size $(\mathrm{mm})$ & $0.5 \times 10^{-3}$ & $0.5 \times 10^{-3}$ & $0.5 \times 10^{-3}$ & 0.005 & 0.05 & 0.1 \\
\hline Degrees of freedom & 5457 & 14160 & 46182 & 35424 & 19446 & 14160 \\
\hline Number of calculations & 102 & 190 & 346 & 234 & 129 & 102 \\
\hline$F_{\text {initiation }}(\mathrm{N})$ & 8809 & 8776 & 8722 & 8875 & 9391 & 9438 \\
\hline$L^{\text {arrest }}(\mathrm{mm})$ & {$[0.7,1.3]$} & {$[0.7,1.2]$} & {$[0.7,1.3]$} & {$[0.7,1.3]$} & {$[1.1,2.0]$} & {$[0.9,1.7]$} \\
\hline
\end{tabular}

Table 1: Crack initiation force and arrest length lower and upper bounds predicted using CC as a function of the minimum mesh size along the crack path in the case of a rhombus hole with $\beta=90 \mathrm{deg}$. angle and $s \times \cos \left(\frac{\beta}{2}\right)=4.95 \mathrm{~mm}$ half-diagonal.

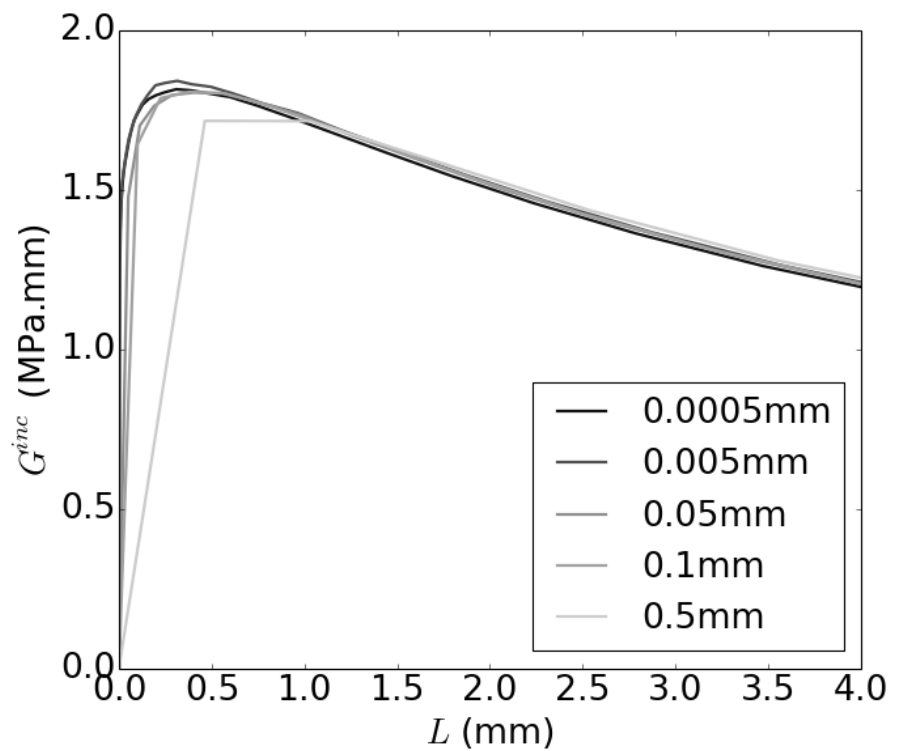

Figure 4: Incremental energy release rate as a function of the crack length obtained for different mesh refinement levels (The size indicated in the legend is the minimum mesh size). 
It is clear that a too coarse mesh does not allow correctly capturing the incremental energy release rate maximum (e.g. for a $0.5 \mathrm{~mm}$ minimum mesh size). It can however be noted that the part of the curve corresponding to crack lengths larger than $1 \mathrm{~mm}$ is well captured whatever the mesh size. Another paramount feature concerns the prediction of the initiation crack length $L^{*}$ (solution of Eq. 4), which adds a second level of mesh refinement. Indeed, it can be shown either analytically [31] or numerically [33] that the initiation length is a fraction of the material characteristic length $L_{\text {mat }}=\frac{E G^{\mathrm{c}}}{\sigma_{c}^{2}}$. Therefore, a fine enough mesh size is required to catch this length when solving Eq. 4. The material under investigation exhibits a characteristic length around $60 \mu \mathrm{m}$, hence leading to initiation lengths as small as $20 \mu \mathrm{m}$. A too large minimum mesh size leads to an erroneous estimate of the crack initiation force and arrest lengths (Table 1). Since the mesh size must not be as small to capture the post-maximum part of the curve as it must be to catch the initiation length, it is possible to unrefine progressively the mesh along the crack path, which makes the computation of the $\mathrm{CC}$ faster. Indeed, it allows decreasing both the number of degrees of freedom and the number of calculations to solve the CC. For instance, using a mesh including a $0.5 \times 10^{-3} \mathrm{~mm}$ minimum size, similar predicted initiation forces and crack arrest lengths are obtained imposing maximum mesh sizes varying from $0.5 \mathrm{~mm}$ to $2 \mathrm{~mm}$. However, increasing the maximum mesh size from $0.5 \mathrm{~mm}$ to $2 \mathrm{~mm}$ allows reducing both the number of calculations needed to solve the CC and the number of degrees of freedom in each calculations (cf. Table 1). In the following, all the calculations for the CC solution have been performed using a $0.5 \mu \mathrm{m}$ minimum mesh size as well as a progressive mesh unrefinement along the crack path to a $2 \mathrm{~mm}$ maximum mesh size, so that around 100 linear elastic calculations are needed to solve the CC.

Fig. 5 shows the crack length as a function of the force predicted using the $\mathrm{CC}$ for a hole with $\beta=90 \mathrm{deg}$. angle and $s \times \cos \frac{\beta}{2}=4.95 \mathrm{~mm}$ half-diagonal. The crack arrest lengths obtained taking into account or not the energy excess available for further propagation of the nucleated crack respectively correspond to the crack arrest upper and lower bounds. Crack propagation (i.e. the variation of $L$ as a function of $F$ after initiation) has been computed using the same incremental algorithm as proposed by Doitrand et al. [10] to study crack propagation in woven composites. At crack initiation, if the energy surplus available for further crack propagation is not used (cf. Fig. 1), the crack reaches its arrest length (i.e the lower bound predicted by the CC) so that $G=G_{c}$ and $\frac{d G}{d L} \leq 0$, corresponding to a stable crack propagation. However, if further crack propagation is allowed by taking into account the energy surplus, the crack arrest length (i.e. the upper bound predicted by the CC) corresponds to a configuration for which $G<G_{c}$. Therefore, a sufficient increase in the loading is necessary for the crack to propagate again (i.e. so that $G=G_{c}$ ). Since except just after initiation (in the case the energy excess is taken into account), no unstable crack propagation phase is observed, similar variations of the crack length as a function of force is obtained whether the external dissipation is taken into account or not [10].

\subsection{CZM implementation}

Contrary to the $\mathrm{CC}$ approach, which requires several linear elastic calculations to be computed, only one non-linear calculation is needed in the case of CZM. The use of CZM requires sufficiently fine mesh discretization so as to accurately represent the process zone ahead of the crack. The order of magnitude of 


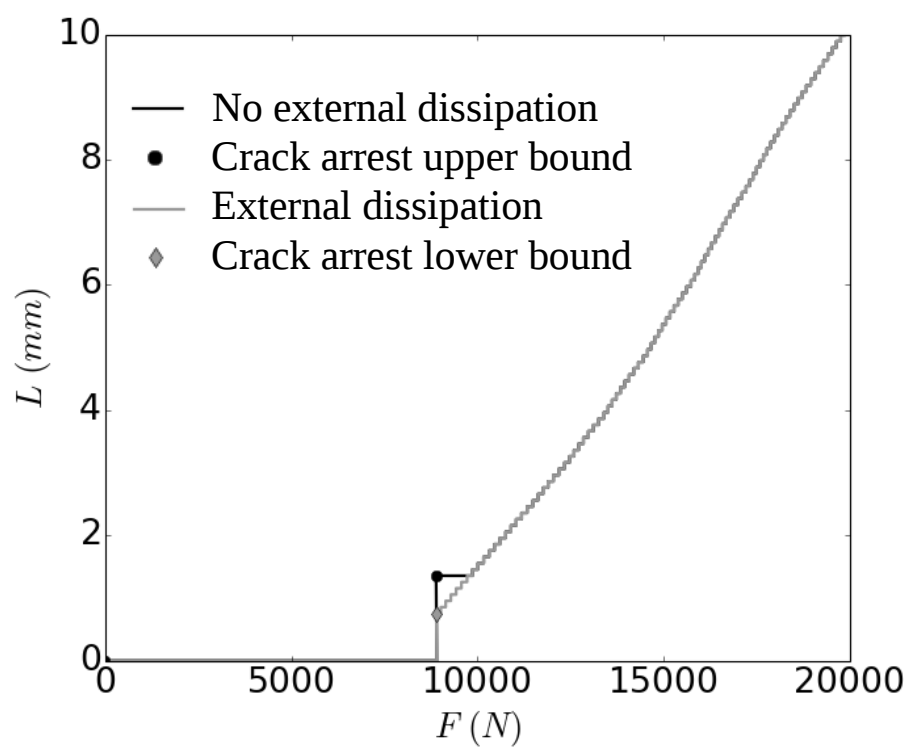

Figure 5: Crack length as a function of force obtained employing the $\mathrm{CC}$ in the case of a hole with $\beta=90$ deg. angle and $s \times \cos \left(\frac{\beta}{2}\right)=4.95 \mathrm{~mm}$ half-diagonal. The initiation crack arrest upper and lower bounds are obtained if the energy surplus at initiation is used (no external dissipation) or not (external dissipation) for further crack propagation.

the process zone length is generally a fraction of the material characteristic length $L_{\text {mat }}[1,4,13,32,39]$. In the present case, the process zone length is $50 \mu \mathrm{m}$, therefore a minimum mesh size of $5 \mu \mathrm{m}$ has been imposed along the crack path so that the process zone contains around 10 elements. The other numerical details of the computations are given in the rest of this section. Fig. 6 shows the crack length as a function of force obtained in the case of a bilinear CZM. The elastic slope of the CZM, whose influence is studied in the following, is set to $K_{C Z M}=10^{8} \mathrm{MPa} / \mathrm{mm}$. Similarly to the CC approach, a crack jump at initiation is obtained. Indeed, just after nucleation, the crack propagates in an unstable manner and directly reaches a length of $0.6 \mathrm{~mm}$ in a really short period (corresponding to an imposed displacement increment lower than $10^{-5} \mathrm{~mm}$ ). This result supports one of the main assumptions made when using the $\mathrm{CC}$, namely the spontaneous crack initiation over a given length. The initiation configuration is described by the force level together with the crack arrest length after the jump. These quantities will be compared to the CC results in next section.

The softening behavior of the CZM may lead to convergence issue in quasi-static numerical simulations $[1,6,14]$. In particular, this is observed in the case of unstable crack propagation, for which solution jumps occur. Several methods exist in order to overcome this problem such as introducing a fictitious viscous regularization $[6,14]$. An other way consists in performing dynamic rather than quasi-static numerical simulations [1], which naturally introduces a relaxation that allows avoiding convergence issues. Such a choice can be justified by the fact that an unstable crack propagation is itself a dynamic process, even under quasi-static loading. All the computations involving CZM have been performed via dynamic numerical simulations, using the PMMA density $\rho=1.18 \times 10^{-3} \mathrm{~g} / \mathrm{mm}^{3}$.

Since we assume a quasi-static loading state in the CC solution (even if crack initiation is considered as a spontaneous and dynamic phenomenon), it is paramount to study the influence of the imposed displacement 


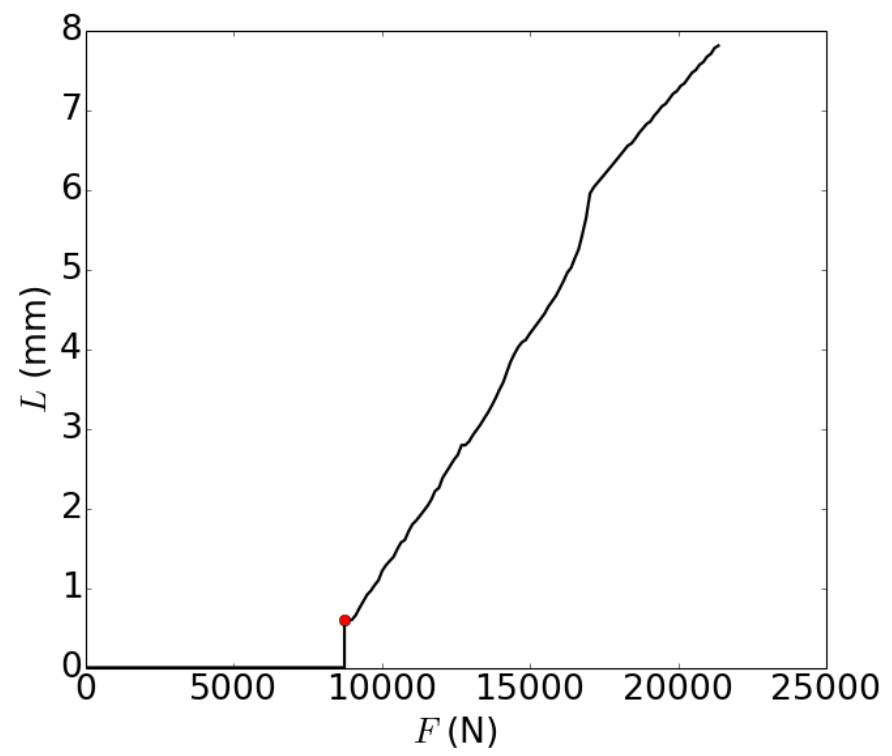

Figure 6: Crack length as a function of force obtained with bilinear CZM in the case of a hole with $\beta=90$ deg. angle and $s \times \cos \left(\frac{\beta}{2}\right)=4.95 \mathrm{~mm}$ half-diagonal.

rate on crack initiation in CZM simulation in order to provide a consistent comparison between both methods. In order to check the overall quasi-static state, several calculations with varying imposed displacement rate have been performed. The initiation force and crack arrest length variations as a function of the imposed displacement rate are shown in Fig. 7. The displacement increment during which the crack jump occurs are summarized in Table 2. The initiation force and the crack arrest length decrease with decreasing displacement rate and reaches a plateau for displacement rates lower than $1 \mathrm{~mm} / \mathrm{s}$, corresponding to an overall quasistatic state. The displacement increment corresponding to crack initiation also decreases with decreasing displacement rate. Therefore, whatever the applied displacement rate, the crack jump occurs within a time increment of $5 \pm 1 \times 10^{-5} \mathrm{~s}$. In the following, a $1 \times 10^{-1} \mathrm{~mm} / \mathrm{s}$ displacement rate has been imposed in simulations involving CZM so that the predicted initiation force and crack arrest length do not depend on the displacement rate.

In CZM models, the elastic part of the traction-opening displacement curve (i.e for $T<\sigma_{c}$ and $\delta<\delta_{0}$ ) introduces an artificial cohesive element stiffness $K_{C Z M}=\frac{\sigma_{c}}{\delta_{0}}$. However, the purpose of the cohesive zone elements is to simulate fracture which means that the stiffness of the cohesive element must be high enough so that it does not affect the overall rigidity of the model [39]. Fig. 8 shows the initiation force and crack arrest length obtained varying the CZM stiffness from $10^{5} \mathrm{MPa} / \mathrm{mm}$ to $10^{10} \mathrm{MPa} / \mathrm{mm}$. Too small values of the CZM stiffness delay crack initiation and lead to a slight underestimate of the crack arrest length. However,

\begin{tabular}{|c|c|c|c|c|c|c|}
\hline Displacement rate $(\mathrm{mm} / \mathrm{s})$ & $1 \times 10^{-2}$ & $1 \times 10^{-1}$ & 1 & 10 & 100 & 1000 \\
\hline Crack jump displacement increment $(\mathrm{mm})$ & $4 \times 10^{-7}$ & $5 \times 10^{-6}$ & $6 \times 10^{-5}$ & $4 \times 10^{-4}$ & $5 \times 10^{-3}$ & $5 \times 10^{-2}$ \\
\hline
\end{tabular}

Table 2: Displacement and time increment during which occurs the initiation crack jump for several imposed displacement rates. 


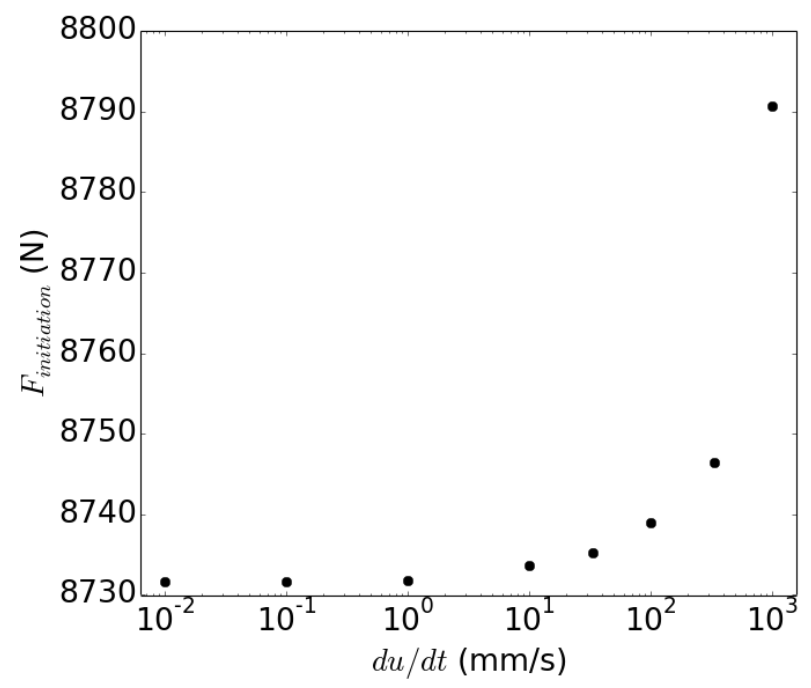

(a)

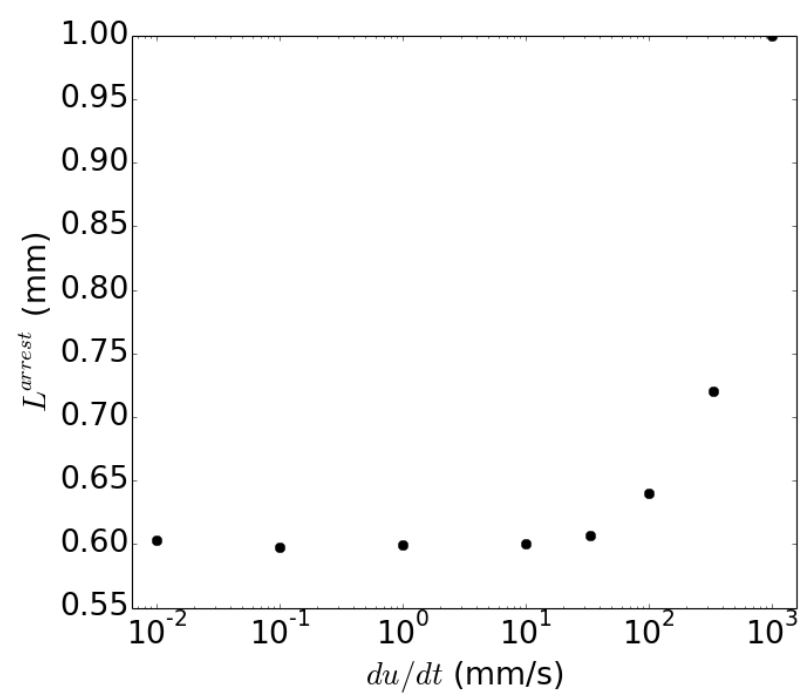

(b)

Figure 7: (a) Initiation force and (b) crack arrest length as a function of the imposed displacement rate in the case of a hole with $\beta=90$ deg. angle and $s \times \cos \left(\frac{\beta}{2}\right)=4.95 \mathrm{~mm}$ half-diagonal using a bilinear CZM model.

similar initiation forces and crack arrest lengths are obtained using a stiffness larger than $10^{7} \mathrm{MPa} / \mathrm{mm}$. In the following, all the calculations have been performed using a CZM stiffness $K_{C Z M}=1 \times 10^{8} \mathrm{MPa} / \mathrm{mm}$. It can be noted that increasing the CZM stiffness also increases the number of iterations and decreases the minimum required time increment (cf. Table 3), as already observed by Turon et al. [39]

Previous analyses concern a bilinear CZM, corresponding to a linear softening part of the traction-opening displacement curve. However, for given fracture parameters $G_{c}$ and $\sigma_{c}$, it is possible to vary the CZM profile, which may influence crack initiation $[1,3]$. Park et al. $[35,36]$ proposed a model that is able to represent numerous CZM profiles by varying a single parameter $\alpha$. A Dugdale-like model is obtained when $\alpha$ tends towards 1 ( $\alpha$ must be strictly larger than 1 for numerical reasons). If $\alpha=2$, a bilinear model is obtained and $\alpha>2$ leads to trilinear-like models. Graphical representations of the traction-opening displacement curve obtained for $\alpha=1.01,2$ and 5 are shown in Fig. 9a. Whatever the CZM profile, the elastic slope for opening displacement lower than the characteristic opening displacement $\delta_{0}$ is also set to $K_{C Z M}=1 \times 10^{8} \mathrm{MPa} / \mathrm{mm}$ so that the overall rigidity of the model is not altered. Keeping the values of $K_{C Z M}, G_{c}$ and $\sigma_{c}$ constant, the only parameter varying when changing the CZM profile is the critical opening displacement $\delta_{c}$, whose value is given as a function of the shape parameter $\alpha$ in Table 4 .

Fig. 9 shows the force and the initiation crack arrest length obtained for different CZM profiles as

\begin{tabular}{|c|c|c|c|c|c|c|}
\hline$K_{C Z M}$ & $1 \times 10^{5}$ & $1 \times 10^{6}$ & $1 \times 10^{7}$ & $1 \times 10^{8}$ & $1 \times 10^{9}$ & $1 \times 10^{10}$ \\
\hline Number of iterations & 155 & 180 & 187 & 206 & 219 & 328 \\
\hline Minimum time increment (s) & $1.2 \times 10^{-7}$ & $1.0 \times 10^{-7}$ & $1.4 \times 10^{-8}$ & $1.5 \times 10^{-9}$ & $4.1 \times 10^{-10}$ & $2.4 \times 10^{-10}$ \\
\hline
\end{tabular}

Table 3: Critical opening displacement of the cohesive zone as a function of the shape parameter $\alpha$. 


\begin{tabular}{|c|c|c|c|c|c|}
\hline$\alpha$ & 1.01 & 1.2 & 2 & 3.5 & 5 \\
\hline$\delta_{\mathrm{c}}(\mathrm{mm})$ & 0.003 & 0.004 & 0.006 & 0.01 & 0.015 \\
\hline
\end{tabular}

Table 4: Critical opening displacement of the cohesive zone as a function of the shape parameter $\alpha$.

a function of the shape parameter. Both crack initiation force and arrest length increases as the CZM profile tends to a Dugdale-like profile. This might be explained by the fact that when the critical opening displacement decreases (i.e. when $\alpha$ decreases), the length on which the material strength $\sigma_{c}$ has to be attained before failure increases (in order to keep a constant fracture energy), which required a larger imposed loading and thus leads to a larger crack arrest length.

\section{Comparison between CC and CZM}

\subsection{Crack initiation prediction}

The initiation forces and crack arrest lengths predicted using CC and CZM are compared in this section for several rhombus hole specimen configuration. The hole angle $\beta$ varies from $70 \mathrm{deg}$. to $110 \mathrm{deg}$. while its half diagonal $s \times \cos \frac{\beta}{2}$ is kept constant. Fig. 10 depicts the initiation force (Fig. 10a) and crack arrest lengths (Fig. 10b) obtained using CC or CZM with various profiles as a function of the hole angle $\beta$. The initiation force decreases with increasing angles. This trends is predicted using both CC or CZM with different profiles. The initiation force predicted using the $\mathrm{CC}$ is comprised between the initiation force obtained with a Dugdale-like $(\alpha=1.01)$ or a trilinear-like CZM $(\alpha=5)$. The differences between the bilinear model and $\mathrm{CC}$ initiation force predictions are around $3 \%$. The crack arrest lengths increase with increasing hole angle. This trend is, once again, predicted using both CZM or CC. The difference between the upper and lower

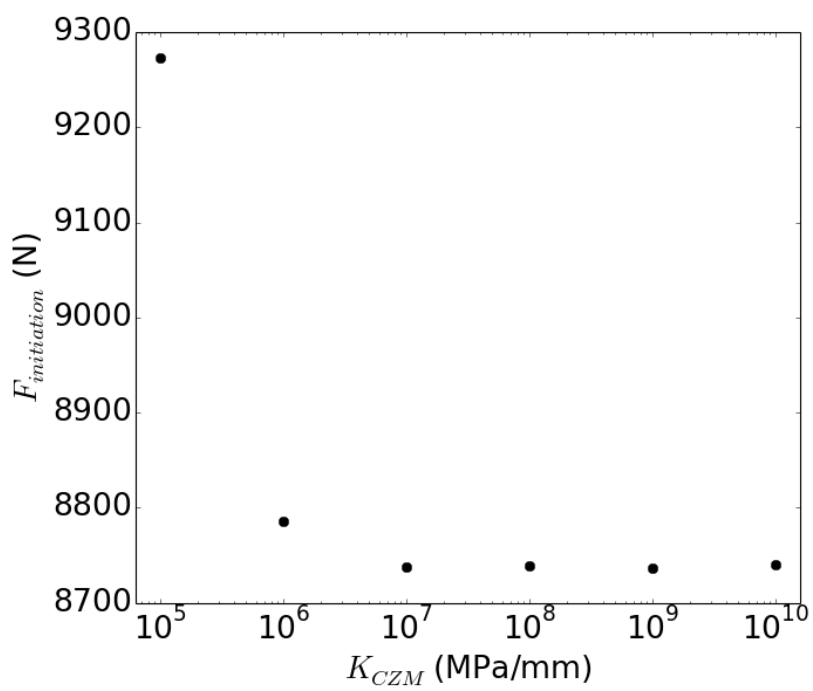

(a)

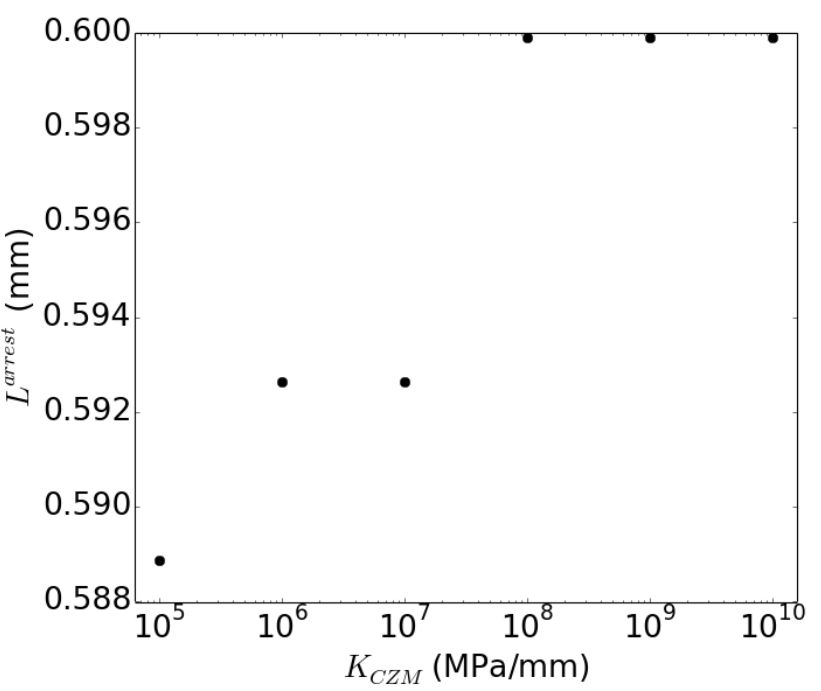

(b)

Figure 8: (a) Initiation force and (b) crack arrest length as a function of the bilinear CZM stiffness in the case of a hole with $\beta=90$ deg. angle and $s \times \cos \left(\frac{\beta}{2}\right)=4.95 \mathrm{~mm}$ half-diagonal. 


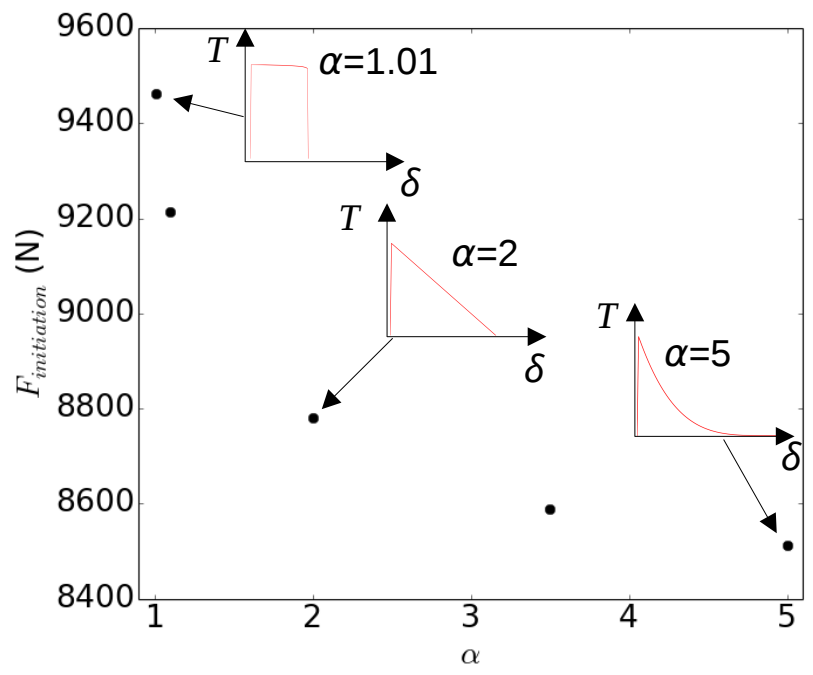

(a)

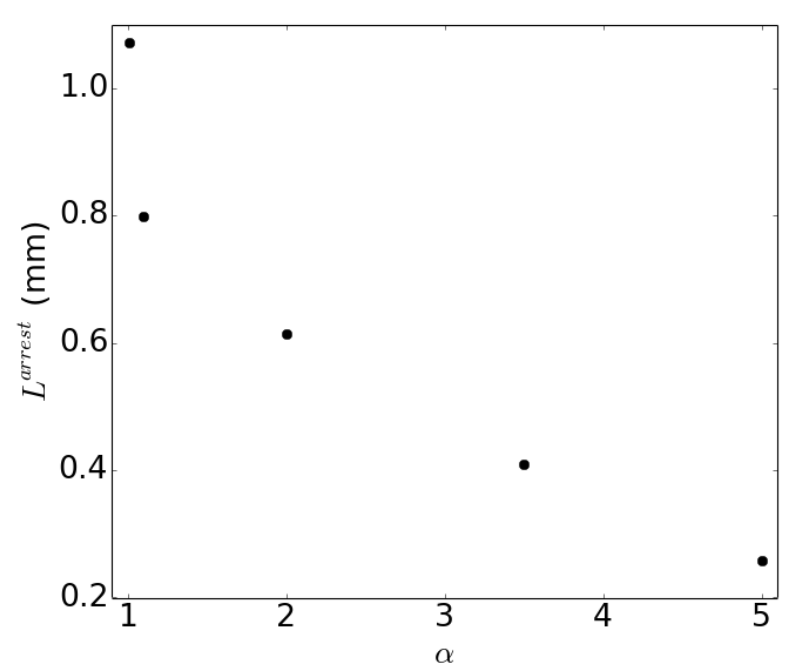

(b)

Figure 9: Initiation (a) force and (b) crack length as a function of the shape parameter $\alpha$ of the CZM.

bounds for crack arrest length increases with increasing hole angle, as well as the range between the crack arrest lengths predicted using different CZM profiles. The range of crack arrest length obtained with different CZM profiles comprises the crack arrest lower bound predicted by the CC, and bilinear CZM results in crack arrest length in the same order of magnitude as the lower bound predicted by the CC.

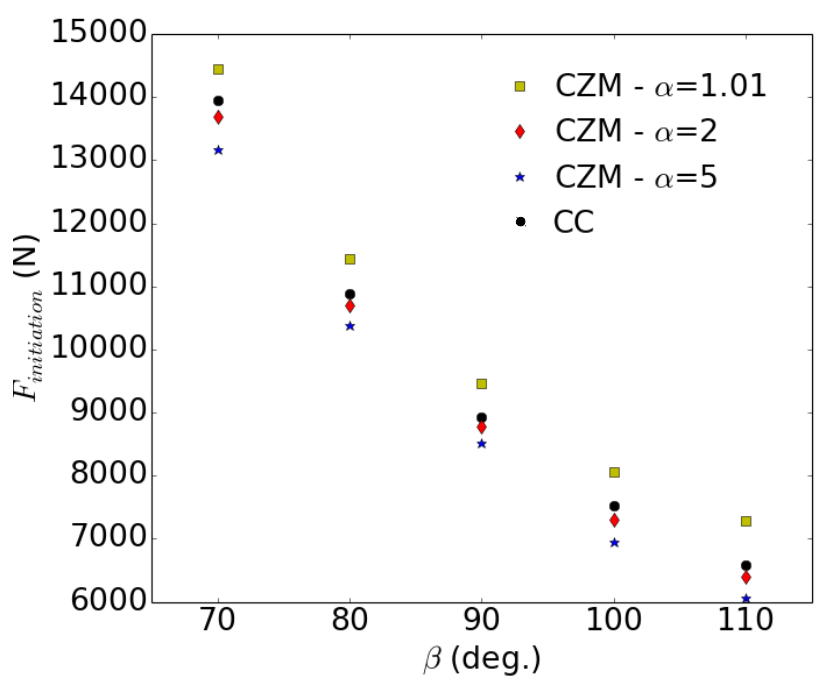

(a)

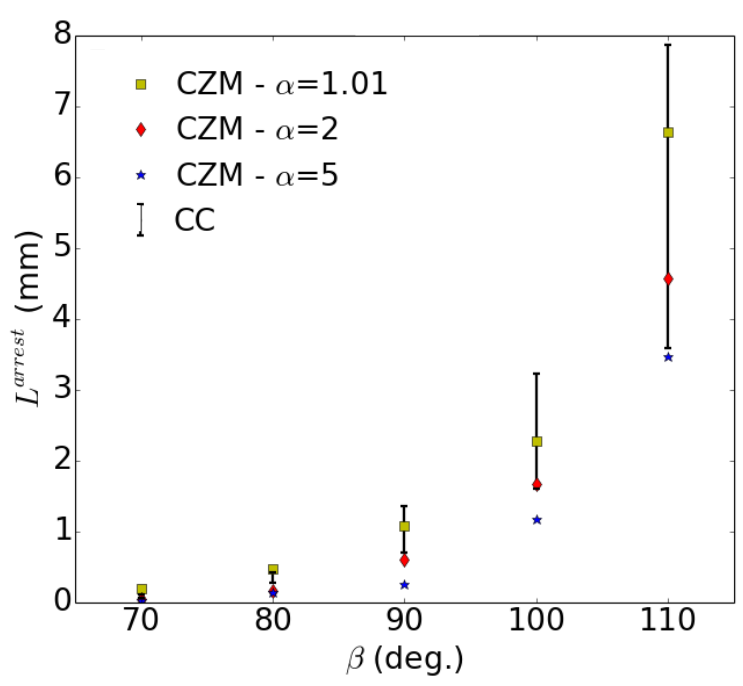

(b)

Figure 10: Initiation (a) force and (b) crack arrest length obtained using CC or CZM with different profiles as a function of rhombus hole angle $\beta$ for a rhombus half diagonal $s \times \cos \left(\frac{\beta}{2}\right)=4.95 \mathrm{~mm}$. 


\subsection{Discussion}

Previous works about the comparison between CC and CZM for crack initiation prediction were mainly focused about confronting the initiation loading predicted by both methods $[7,15,19,32,37]$. In all of these studies, only one CZM was employed so that varying the CZM profile was not accounted for. In the present study, we highlight that the choice of the CZM has a non-negligible influence on the predicted initiation force. For instance, differences up to 15\% (cf. Fig. 10a) are obtained between the initiation force predicted using a Dudgale-like or trilinear-like CZM.

An important aspect that can also be compared is the computational cost of both approaches. As regards $\mathrm{CC}$, following the mesh analysis presented in section 4.2, the whole CC analysis (generation of the model, calculations and post-treatment) requires around $15 \mathrm{~min}$ to be completed. Since a sufficiently refined mesh is required over the whole crack path when using CZM ( $c f$. section 4.3), meshes with around 500000 degrees of freedom are obtained, corresponding to calculations duration longer than $10 \mathrm{H}$. Such a refinement along the crack path is required if the crack arrest length is not known, since large crack arrest lengths (e.g., for $\beta=110$ deg.) can be obtained. The calculation time can be reduced to around $1 \mathrm{H}$ in the case of smaller crack arrest lengths (e.g., for $\beta=70$ deg.) by unrefining the mesh along the crack path at a certain distance of the crack arrest length and only considering the initiation phase, which is only possible if the crack arrest length is known a priori.

One of the main advantages of the CC compared to CZM is that the toughness and strength values are only implemented during the post-processing step. Therefore, provided that the mesh size is small enough to correctly represent $G^{i n c}$ and capture the initiation length $L^{*}$ ( $c f$. section 4.2), the FE calculations with different crack lengths can be computed once to obtain initiation force and crack arrest length for different values of $G_{c}$ and $\sigma_{c}$. This is particularly convenient in the case where these fracture parameters are unknown and have to be determined by inverse confrontation to experimental results [11, 12]. This is not the case employing CZM, which requires several calculations with varying $G_{c}$ and $\sigma_{c}$ to be computed. Conversely, in the case where several crack configurations have to be studied for given fracture parameters, the number of calculations to perform in order to solve the CC exponentially increases with the number of parameters describing the crack [9]. In this case, the CZM can be a powerfull approach since it allows taking into account, for instance, different crack paths in a single computation, obviously leading to more costly computations due to an increase in the number of dof and more restrictive convergence conditions.

\section{Conclusion}

Crack nucleation in rhombus hole specimens under compression modeled using either CZM or CC leads to the conclusion that at initiation, there is a crack jump to a given length at a certain loading. Even under overall quasi-static loading conditions, the crack jump is a dynamic and instantaneous phenomenon. The CC allows determining the initiation loading as well as a lower and upper bounds for crack arrest by considering the unstable crack propagation just after initiation, taking into account or not the energy surplus for further 
crack propagation. The initiation loading and the crack arrest length can also be determined using CZM. For fixed fracture parameters $G_{c}$ and $\sigma_{c}$, both quantities depends on the CZM profile.

$\mathrm{CC}$ and $\mathrm{CZM}$ predict similar trends of the initiation force and crack arrest length as a function of the rhombus hole angle. With increasing hole angle, the initiation force decreases whereas the crack arrest length increases. For various geometrical configurations, the initiation force predicted using the CC lies within the range of those obtained with different CZM profiles. Similarly, the range of crack arrest lengths obtained with different CZM profiles comprises the crack arrest length lower bound obtained with the CC. For the configurations under investigation, similar results are obtained with the CC and bilinear CZM. Confronting CZM and CC appears to be a way to identify the CZM profile for given material parameters.

CZM requires a sufficiently refined mesh over the whole crack path in order to correctly account for the process zone ahead of the crack tip. For CC computation, the mesh must be sufficiently fine to catch the initiation length and correctly describe the incremental energy release rate but it is possible to unrefine it along the crack path to decrease both the number of calculations and the number of degrees of freedom in each calculations (and thus to save computational time). In both cases, the minimum mesh size can be related to the material characteristic length $\frac{E G_{c}}{\sigma_{c}^{2}}$. In the present study, the CC appears to be more computationally efficient that CZM. A main advantage of CC approach compared to CZM is that all the calculations can be done without assuming any values of $G_{c}$ and $\sigma_{c}$ (except in order to ensure a sufficiently fine minimum mesh size), that are only implemented while in the calculation post-treatment. Therefore, this approach can be really convenient in order to determine unknown fracture parameters by inverse confrontation to experimental results $[11,12,23]$. On the contrary, if many possible crack configurations have to be studied, the number of calculations rapidly increases using $\mathrm{CC}$, therefore CZM might be preferred in this case.

The present analysis exclusively concerns the comparison of two numerical methods. Future work will cover an experimental analysis of crack initiation in rhombus hole specimens so as to evaluate the validity of the models, especially regarding the predicted spontaneous crack initiation. 


\section{References}

[1] Acary, V., Monerie, Y., 2006. Nonsmooth fracture dynamics using a cohesive zone approach. Research Report RR-6032, INRIA.

[2] Alfano, G., Crisfield, MA., 2001. Finite element interface models for the delamination analysis of laminated composites: mechanical and computational issues. Int. J. Numer. Meth. Engng 50, 17011736.

[3] Alfano, G., 2006. On the influence of the shape of the interface law on the application of cohesive-zone models. Compos. Sci. Tech 66, 723-730.

[4] Barenblatt, G.I. ,1959. The formation of equilibrium cracks during brittle fracture: general ideas and hypotheses. Axially-symmetric Cracks PMM 23, 622636.

[5] Carrère, N., Martin, E., Leguillon, D., 2015. Comparison between models based on a coupled criterion for the prediction of the failure of adhesively bonded joint. Engng. Fract. Mech. 138, 185-201.

[6] Chaboche, J.L., Feyel, F., Monerie, Y., 2001. Interface debonding model: a viscous regularization with a limited rate dependency. Int. J. Sol. Struct. 38, 3127-3160.

[7] Cornetti, P., Sapora, A., Carpinteri, A., 2016. Short cracks and V-notches: Finite Fracture Mechanics vs. Cohesive Crack Model. Engng. Fract. Mech. 168, 2-12.

[8] Cornetti, P., Munũnoz-Reja, M., Sapora, A., Carpinteri, A. Finite Fracture Mechanics and cohesive crack model: weight functions vs. cohesive laws. Int. J. Sol. Struct. doi:10.1016/j.ijsolstr.2018.08.003.

[9] Doitrand, A., Fagiano, C., Carrère, N., Chiaruttini, V., Hirsekorn, M., 2017. Damage onset modeling in woven composites based on a coupled stress and energy criterion. Engng. Fract. Mech. 169, 189-200.

[10] Doitrand, A., Fagiano, C., Hild F., Chiaruttini, V., Mavel A., Hirsekorn, M., 2017. Mesoscale analysis of damage growth in woven composites Compos Part A. 96, 77-88.

[11] Doitrand, A., Leguillon, D, 2018. 3D application of the coupled criterion to crack initiation prediction in epoxy/aluminum specimens under four point bending. Int. J. Sol. Struct 143, 175-182.

[12] Doitrand, A., Leguillon, D, 2018. Comparison between 2D and 3D applications of the coupled criterion to crack initiation prediction in scarf adhesive joints. Int. J. Adh. Adh. 85, 69-76.

[13] Dugdale, D.S., 1960. Yielding of steel sheets containing slits. J. Mech. Phys. Sol 8,100104.

[14] Gao, Y.F., Bower, A.F., A simple technique for avoiding convergence problems in finite element simulations of crack nucleation and growth on cohesive interfaces. Modelling Simul. Mater. Sci. Eng. 12, 453-463.

[15] García, I.G., Paggi M., Mantič V., 2014. Fiber-size effects on the onset of fibermatrix debonding under transverse tension: A comparison between cohesive zone and finite fracture mechanics models. Engng. Fract. Mech. 115, 96-110. 
[16] García, I.G., Mantič, V., Graciani, E., 2015. A model for the prediction of debond onset in sphericalparticle-reinforced composites under tension. Application of a coupled stress and energy criterion. Compos. Sci. Technol. 106, 60-67.

[17] García, I.G., Carter, B.J., Ingraffea, A.R., Mantič V., 2016. A numerical study of transverse cracking in cross-ply laminates by 3D finite fracture mechanics. Compos. Part B 95, 475-487.

[18] Hashin, Z.,1996. Finite thermoelastic fracture criterion with application to laminate cracking analysis. J. Mech. Phys. Solids 44(7), 11291145.

[19] Henninger, C., Leguillon, D., Martin, E., 2007. Crack initiation at a V-notch-comparison between a brittle fracture criterion and the Dugdale cohesive model. C. R. Mecanique 335, 388-393.

[20] Leguillon, D., 2002. Strength or toughness? A criterion for crack onset at a notch. Eur. J. Mech. A/Solids 21 (1), 61-72.

[21] Leguillon, D., Yosibash, Z., 2003. Crack onset at a v-notch. Influence of the notch tip radius. Int. J. Fract. 122(1-2), 1-21.

[22] Leguillon, D. , Quesada, D. , Putot, C. , Martin, E. , 2007. Size effects for crack initiation at blunt notches or cavities. Engng. Fract. Mech. 74, 24202436 .

[23] Leguillon, D., 2014. An attempt to extend the $2 \mathrm{~d}$ coupled criterion for crack nucleation in brittle materials to the 3d case. Theor. Appl. Fract. Mech. 74, 717 .

[24] Leguillon, D., Martin, E., Seveček, O., Bermejo, R. (2015). Application of the coupled stress-energy criterion to predict the fracture behaviour of layered ceramics designed with internal compressive stresses. Eur. J. Mech. A/Solids, 54, 94-104.

[25] Leguillon, D., Yosibash, Z., 2017. Failure initiation at V-notch tips in quasi-brittle materials. Int. J. Solids Structures, 122-123, 1-13.

[26] Leguillon, D., Martin, E., Seveček, O., Bermejo R, 2018. What is the tensile strength of a ceramic to be used in numerical models for predicting crack initiation? Int. J. Fract. 212(1), 89-103.

[27] Mantič, V., 2009. Interface crack onset at a circular cylindrical inclusion under a remote transverse tension. Application of a coupled stress and energy criterion. Int. J. Sol. Struct. 46, 1287-1304.

[28] Mantič, V., García, I.G., 2012. Crack onset and growth at the fibre-matrix interface under a remote biaxial transverse load. Application of a coupled stress and energy criterion. Int. J. Solids Structures 49, 2273-2290.

[29] Martin, E., Leguillon, D., 2004. Energetic conditions for interfacial failure in the vicinity of a matrix crack in brittle matrix composites. Int. J. Solids Structures 41, 6937-6948. 
[30] Martin, E., Poitou, B., Leguillon, D., Gatt, J.M., 2008. Competition between deflection and penetration at an interface in the vicinity of a main crack. Int. J. Fract. 151(2), 247268.

[31] Martin, E., Leguillon, D., Carrère, N., 2012. A coupled strength and toughness criterion for the prediction of the open hole tensile strength of a composite plate. Int. J. Solids and Structures 49 (26), 3915-3922.

[32] Martin, E., Vandellos, T., Leguillon, D., Carrère, N., 2016. Initiation of edge debonding: coupled criterion versus cohesive zone model. International Journal of Fracture. 199, 157168.

[33] Martin, E., Leguillon, D., Seveček, O., Bermejo, R., 2018. Understanding the tensile strength of ceramics in the presence of small critical flaws. Engng. Fract. Mech. doi:10.1016/j.engfracmech.2018.06.021.

[34] Nairn, J.A., 2000. Exact and variational theorems for fracture mechanics of composites with residual stresses, traction-loaded cracks, and imperfect interfaces. Int. J. Fract. 105, 243-271.

[35] Park, K., Paulino, G.H., Roesler, J.R., 2009. A unified potential-based cohesive model of mixed-mode fracture. J. Mech. Phys. Sol. 57, 891-908.

[36] Park, K., Paulino, G.H., 2012. Computational implementation of the PPR potential-based cohesive model in ABAQUS: Educational perspective. Engng. Fract. Mech. 93, 239-262.

[37] Poitou, B., Martin, E., Carrère, N., Leguillon, D., Gatt, J.M, 2007. Amorçage de fissure au voisinage des interfaces fibre/matrice : comparaison dun critère mixte et des modèles de zone cohésive. $18^{\text {eme }}$ Congrè Français de Mécanique, Grenoble, 27-31 August 2007.

[38] Stein, N., Weigraeber, P., Becker, W., 2015. A model for brittle failure in adhesive lap joints of arbitrary joint configuration. Compos Struct 133, 707-718.

[39] Turon, A., Dávila, C.G., Camanho, P.P., Costa, J., 2007. An engineering solution for mesh size effects in the simulation of delamination using cohesive zone models. Engng. Fract. Mech. 74, 1665-1682.

[40] Tvergaard, V., Hutchinson, J.W., 1992. The relation between crack growth resistance and fracture process parameters in elastic-plastic solids. J. Mech. Phys. Sol. 40,13771397.

[41] Weißgraeber, P., Leguillon, D., Becker, W., 2016. A review of Finite Fracture Mechanics: crack initiation at singular and non-singular stress raisers. Archive Appl. Mech. 86 (1-2), 375401.

[42] Weissgraeber, P., Hell, S., Becker, W., 2016. Crack nucleation in negative geometries. Engng. Fract. Mech. 168, 93-104.

[43] Xu, X., Needleman, A., 1994. Numerical simulations of fast crack growth in brittle solids. J. Mech. Phys. Sol. 42, 13971434. 